\title{
Iterative common solutions for monotone inclusion problems, fixed point problems and equilibrium problems
}

\author{
Wataru Takahashi ${ }^{1}$, Ngai-Ching Wong ${ }^{2}$ and Jen-Chih Yao $^{3 *}$
}

\section{"Correspondence:}

yaojc@kmu.edu.tw

${ }^{3}$ Center for General Education,

Kaohsiung Medical University,

Kaohsiung, 80702, Taiwan

Full list of author information is

available at the end of the article

\begin{abstract}
Let $H$ be a real Hilbert space, and let $C$ be a nonempty closed convex subset of $H$. Let $\alpha>0$, and let $A$ be an $\alpha$-inverse strongly-monotone mapping of $C$ into $H$. Let $T$ be a generalized hybrid mapping of $C$ into $H$. Let $B$ and $W$ be maximal monotone operators on $H$ such that the domains of $B$ and $W$ are included in $C$. Let $0<k<1$, and let $g$ be a $k$-contraction of $H$ into itself. Let $V$ be a $\bar{\gamma}$-strongly monotone and $L$-Lipschitzian continuous operator with $\bar{\gamma}>0$ and $L>0$. Take $\mu, \gamma \in \mathbb{R}$ as follows:

$$
0<\mu<\frac{2 \bar{\gamma}}{L^{2}}, \quad 0<\gamma<\frac{\bar{\gamma}-\frac{L^{2} \mu}{2}}{k} .
$$
\end{abstract}

Suppose that $F(T) \cap(A+B)^{-1} 0 \cap W^{-1} 0 \neq \emptyset$, where $F(T)$ and $(A+B)^{-1} 0, W^{-1} 0$ are the set of fixed points of $T$ and the sets of zero points of $A+B$ and $W$, respectively. In this paper, we prove a strong convergence theorem for finding a point $z_{0}$ of $F(T) \cap(A+B)^{-1} 0 \cap W^{-1} 0$, where $z_{0}$ is a unique fixed point of $P_{F(T) \cap(A+B)^{-1} 0 \cap W^{-1} 0}(I-V+\gamma g)$. This point $z_{0} \in F(T) \cap(A+B)^{-1} 0 \cap W^{-1} 0$ is also a unique solution of the variational inequality

$$
\left\langle(V-\gamma g) z_{0}, q-z_{0}\right\rangle \geq 0, \quad \forall q \in F(T) \cap(A+B)^{-1} 0 \cap W^{-1} 0 .
$$

Using this result, we obtain new and well-known strong convergence theorems in a Hilbert space. In particular, we solve a problem posed by Kurokawa and Takahashi (Nonlinear Anal. 73:1562-1568, 2010).

MSC: $47 \mathrm{H} 05 ; 47 \mathrm{H} 10 ; 58 \mathrm{E} 35$

Keywords: maximal monotone operator; resolvent; inverse-strongly monotone mapping; generalized hybrid mapping; fixed point; iteration procedure; equilibrium problem

\section{Introduction}

Let $H$ be a real Hilbert space, and let $C$ be a nonempty closed convex subset of $H$. Let $\mathbb{N}$ and $\mathbb{R}$ be the sets of positive integers and real numbers, respectively. A mapping $T: C \rightarrow H$ is called generalized hybrid [1] if there exist $\alpha, \beta \in \mathbb{R}$ such that

$$
\alpha\|T x-T y\|^{2}+(1-\alpha)\|x-T y\|^{2} \leq \beta\|T x-y\|^{2}+(1-\beta)\|x-y\|^{2}
$$

(c) 2012 Takahashi et al.; licensee Springer. This is an Open Access article distributed under the terms of the Creative Commons Attribution License (http://creativecommons.org/licenses/by/2.0), which permits unrestricted use, distribution, and reproduction in any medium, provided the original work is properly cited. 
for all $x, y \in C$. We call such a mapping an $(\alpha, \beta)$-generalized hybrid mapping. Kocourek, Takahashi and Yao [1] proved a fixed point theorem for such mappings in a Hilbert space. Furthermore, they proved a nonlinear mean convergence theorem of Baillon's type [2] in a Hilbert space. Notice that the mapping above covers several well-known mappings. For example, an $(\alpha, \beta)$-generalized hybrid mapping $T$ is nonexpansive for $\alpha=1$ and $\beta=0$, i.e.,

$$
\|T x-T y\| \leq\|x-y\|, \quad \forall x, y \in C .
$$

It is also nonspreading [3, 4] for $\alpha=2$ and $\beta=1$, i.e.,

$$
2\|T x-T y\|^{2} \leq\|T x-y\|^{2}+\|T y-x\|^{2}, \quad \forall x, y \in C .
$$

Furthermore, it is hybrid [5] for $\alpha=\frac{3}{2}$ and $\beta=\frac{1}{2}$, i.e.,

$$
3\|T x-T y\|^{2} \leq\|x-y\|^{2}+\|T x-y\|^{2}+\|T y-x\|^{2}, \quad \forall x, y \in C .
$$

We can also show that if $x=T x$, then for any $y \in C$,

$$
\alpha\|x-T y\|^{2}+(1-\alpha)\|x-T y\|^{2} \leq \beta\|x-y\|^{2}+(1-\beta)\|x-y\|^{2},
$$

and hence $\|x-T y\| \leq\|x-y\|$. This means that an $(\alpha, \beta)$-generalized hybrid mapping with a fixed point is quasi-nonexpansive. The following strong convergence theorem of Halpern's type [6] was proved by Wittmann [7]; see also [8].

Theorem 1 Let $C$ be a nonempty closed convex subset of $H$, and let $T$ be a nonexpansive mapping of $C$ into itself with $F(T) \neq \emptyset$. For any $x_{1}=x \in C$, define a sequence $\left\{x_{n}\right\}$ in $C$ by

$$
x_{n+1}=\alpha_{n} x+\left(1-\alpha_{n}\right) T x_{n}, \quad \forall n \in \mathbb{N},
$$

where $\left\{\alpha_{n}\right\} \subset(0,1)$ satisfies

$$
\lim _{n \rightarrow \infty} \alpha_{n}=0, \quad \sum_{n=1}^{\infty} \alpha_{n}=\infty \quad \text { and } \quad \sum_{n=1}^{\infty}\left|\alpha_{n}-\alpha_{n+1}\right|<\infty .
$$

Then $\left\{x_{n}\right\}$ converges strongly to a fixed point of $T$.

Kurokawa and Takahashi [9] also proved the following strong convergence theorem for nonspreading mappings in a Hilbert space; see also Hojo and Takahashi [10] for generalized hybrid mappings.

Theorem 2 Let $C$ be a nonempty closed convex subset of a real Hilbert space H. Let $T$ be a nonspreading mapping of $C$ into itself. Let $u \in C$ and define two sequences $\left\{x_{n}\right\}$ and $\left\{z_{n}\right\}$ in $C$ as follows: $x_{1}=x \in C$ and

$$
\left\{\begin{array}{l}
x_{n+1}=\alpha_{n} u+\left(1-\alpha_{n}\right) z_{n}, \\
z_{n}=\frac{1}{n} \sum_{k=0}^{n-1} T^{k} x_{n}
\end{array}\right.
$$


for all $n=1,2, \ldots$, where $\left\{\alpha_{n}\right\} \subset(0,1), \lim _{n \rightarrow \infty} \alpha_{n}=0$ and $\sum_{n=1}^{\infty} \alpha_{n}=\infty$. If $F(T)$ is nonempty, then $\left\{x_{n}\right\}$ and $\left\{z_{n}\right\}$ converge strongly to $P u$, where $P$ is the metric projection of $H$ onto $F(T)$.

Remark We do not know whether Theorem 1 for nonspreading mappings holds or not; see [9] and [10].

In this paper, we provide a strong convergence theorem for finding a point $z_{0}$ of $F(T) \cap$ $(A+B)^{-1} 0 \cap W^{-1} 0$ such that it is a unique fixed point of

$$
P_{F(T) \cap(A+B)^{-1} 0 \cap W^{-1} 0}(I-V+\gamma g)
$$

and a unique solution of the variational inequality

$$
\left\langle(V-\gamma g) z_{0}, q-z_{0}\right\rangle \geq 0, \quad \forall q \in F(T) \cap(A+B)^{-1} 0 \cap W^{-1} 0,
$$

where $T, A, B, W, g$ and $V$ denote a generalized hybrid mapping of $C$ into $H$, an $\alpha$-inverse strongly-monotone mapping of $C$ into $H$ with $\alpha>0$, maximal monotone operators on $H$ such that the domains of $B$ and $W$ are included in $C$, a $k$-contraction of $H$ into itself with $0<k<1$ and a $\bar{\gamma}$-strongly monotone and $L$-Lipschitzian continuous operator with $\bar{\gamma}>0$ and $L>0$, respectively. Using this result, we obtain new and well-known strong convergence theorems in a Hilbert space. In particular, we solve a problem posed by Kurokawa and Takahashi [9].

\section{Preliminaries}

Let $H$ be a real Hilbert space with inner product $\langle\cdot, \cdot\rangle$ and norm $\|\cdot\|$, respectively. When $\left\{x_{n}\right\}$ is a sequence in $H$, we denote the strong convergence of $\left\{x_{n}\right\}$ to $x \in H$ by $x_{n} \rightarrow x$ and the weak convergence by $x_{n} \rightarrow x$. We have from [11] that for any $x, y \in H$ and $\lambda \in \mathbb{R}$,

$$
\|x+y\|^{2} \leq\|x\|^{2}+2\langle y, x+y\rangle
$$

and

$$
\|\lambda x+(1-\lambda) y\|^{2}=\lambda\|x\|^{2}+(1-\lambda)\|y\|^{2}-\lambda(1-\lambda)\|x-y\|^{2} .
$$

Furthermore, we have that for $x, y, u, v \in H$,

$$
2\langle x-y, u-v\rangle=\|x-v\|^{2}+\|y-u\|^{2}-\|x-u\|^{2}-\|y-v\|^{2} .
$$

All Hilbert spaces satisfy Opial's condition, that is,

$$
\liminf _{n \rightarrow \infty}\left\|x_{n}-u\right\|<\liminf _{n \rightarrow \infty}\left\|x_{n}-v\right\|
$$

if $x_{n} \rightarrow u$ and $u \neq v$; see [12]. Let $C$ be a nonempty closed convex subset of a Hilbert space $H$, and let $T: C \rightarrow H$ be a mapping. We denote by $F(T)$ the set of fixed points for $T$. A mapping $T: C \rightarrow H$ is called quasi-nonexpansive if $F(T) \neq \emptyset$ and $\|T x-y\| \leq\|x-y\|$ 
for all $x \in C$ and $y \in F(T)$. If $T: C \rightarrow H$ is quasi-nonexpansive, then $F(T)$ is closed and convex; see [13]. For a nonempty closed convex subset $C$ of $H$, the nearest point projection of $H$ onto $C$ is denoted by $P_{C}$, that is, $\left\|x-P_{C} x\right\| \leq\|x-y\|$ for all $x \in H$ and $y \in C$. Such $P_{C}$ is called the metric projection of $H$ onto $C$. We know that the metric projection $P_{C}$ is firmly nonexpansive; $\left\|P_{C} x-P_{C} y\right\|^{2} \leq\left\langle P_{C} x-P_{C} y, x-y\right\rangle$ for all $x, y \in H$. Furthermore, $\left\langle x-P_{C} x, y-P_{C} x\right\rangle \leq 0$ holds for all $x \in H$ and $y \in C$; see [14]. The following result is in [15].

Lemma 3 Let $H$ be a Hilbert space, and let $C$ be a nonempty closed convex subset of H. Let $T: C \rightarrow H$ be a generalized hybrid mapping. Suppose that there exists $\left\{x_{n}\right\} \subset C$ such that $x_{n} \rightarrow z$ and $x_{n}-T x_{n} \rightarrow 0$. Then $z \in F(T)$.

Let $B$ be a mapping of $H$ into $2^{H}$. The effective domain of $B$ is denoted by $D(B)$, that is, $D(B)=\{x \in H: B x \neq \emptyset\}$. A multi-valued mapping $B$ is said to be a monotone operator on $H$ if $\langle x-y, u-v\rangle \geq 0$ for all $x, y \in D(B), u \in B x$, and $v \in B y$. A monotone operator $B$ on $H$ is said to be maximal if its graph is not properly contained in the graph of any other monotone operator on $H$. For a maximal monotone operator $B$ on $H$ and $r>0$, we may define a single-valued operator $J_{r}=(I+r B)^{-1}: H \rightarrow D(B)$, which is called the resolvent of $B$ for $r$. We denote by $A_{r}=\frac{1}{r}\left(I-J_{r}\right)$ the Yosida approximation of $B$ for $r>0$. We know from [8] that

$$
A_{r} x \in B J_{r} x, \quad \forall x \in H, r>0 .
$$

Let $B$ be a maximal monotone operator on $H$, and let $B^{-1} 0=\{x \in H: 0 \in B x\}$. It is known that $B^{-1} 0=F\left(J_{r}\right)$ for all $r>0$ and the resolvent $J_{r}$ is firmly nonexpansive, i.e.,

$$
\left\|J_{r} x-J_{r} y\right\|^{2} \leq\left\langle x-y, J_{r} x-J_{r} y\right\rangle, \quad \forall x, y \in H .
$$

We also know the following lemma from [16].

Lemma 4 Let $H$ be a real Hilbert space, and let B be a maximal monotone operator on $H$. For $r>0$ and $x \in H$, define the resolvent $J_{r} x$. Then the following holds:

$$
\frac{s-t}{s}\left\langle J_{s} x-J_{t} x, J_{s} x-x\right\rangle \geq\left\|J_{s} x-J_{t} x\right\|^{2}
$$

for all $s, t>0$ and $x \in H$.

From Lemma 4, we have that

$$
\left\|J_{\lambda} x-J_{\mu} x\right\| \leq(|\lambda-\mu| / \lambda)\left\|x-J_{\lambda} x\right\|
$$

for all $\lambda, \mu>0$ and $x \in H$; see also $[14,17]$. To prove our main result, we need the following lemmas.

Lemma 5 ([18]; see also [19]) Let $\left\{s_{n}\right\}$ be a sequence of nonnegative real numbers, let $\left\{\alpha_{n}\right\}$ be a sequence of $[0,1]$ with $\sum_{n=1}^{\infty} \alpha_{n}=\infty$, let $\left\{\beta_{n}\right\}$ be a sequence of nonnegative real numbers 
with $\sum_{n=1}^{\infty} \beta_{n}<\infty$, and let $\left\{\gamma_{n}\right\}$ be a sequence of real numbers with $\lim _{\sup _{n \rightarrow \infty}} \gamma_{n} \leq 0$. Suppose that

$$
s_{n+1} \leq\left(1-\alpha_{n}\right) s_{n}+\alpha_{n} \gamma_{n}+\beta_{n}
$$

for all $n=1,2, \ldots$. Then $\lim _{n \rightarrow \infty} s_{n}=0$.

Lemma 6 ([20]) Let $\left\{\Gamma_{n}\right\}$ be a sequence of real numbers that does not decrease at infinity in the sense that there exists a subsequence $\left\{\Gamma_{n_{i}}\right\}$ of $\left\{\Gamma_{n}\right\}$ which satisfies $\Gamma_{n_{i}}<\Gamma_{n_{i}+1}$ for all $i \in \mathbb{N}$. Define the sequence $\{\tau(n)\}_{n \geq n_{0}}$ of integers as follows:

$$
\tau(n)=\max \left\{k \leq n: \Gamma_{k}<\Gamma_{k+1}\right\},
$$

where $n_{0} \in \mathbb{N}$ such that $\left\{k \leq n_{0}: \Gamma_{k}<\Gamma_{k+1}\right\} \neq \emptyset$. Then the following hold:

(i) $\tau\left(n_{0}\right) \leq \tau\left(n_{0}+1\right) \leq \cdots$ and $\tau(n) \rightarrow \infty$;

(ii) $\Gamma_{\tau(n)} \leq \Gamma_{\tau(n)+1}$ and $\Gamma_{n} \leq \Gamma_{\tau(n)+1}, \forall n \in \mathbb{N}$.

\section{Strong convergence theorems}

Let $H$ be a real Hilbert space. A mapping $g: H \rightarrow H$ is a contraction if there exists $k \in(0,1)$ such that $\|g(x)-g(y)\| \leq k\|x-y\|$ for all $x, y \in H$. We call such a mapping $g$ a $k$-contraction. A nonlinear operator $V: H \rightarrow H$ is called strongly monotone if there exists $\bar{\gamma}>0$ such that $\langle x-y, V x-V y\rangle \geq \bar{\gamma}\|x-y\|^{2}$ for all $x, y \in H$. Such $V$ is also called $\bar{\gamma}$-strongly monotone. A nonlinear operator $V: H \rightarrow H$ is called Lipschitzian continuous if there exists $L>0$ such that $\|V x-V y\| \leq L\|x-y\|$ for all $x, y \in H$. Such $V$ is also called $L$-Lipschitzian continuous. We know the following three lemmas in a Hilbert space; see Lin and Takahashi [21].

Lemma 7 ([21]) Let $H$ be a Hilbert space, and let $V$ be a $\bar{\gamma}$-strongly monotone and $L$ Lipschitzian continuous operator on $H$ with $\bar{\gamma}>0$ and $L>0$. Let $t>0$ satisfy $2 \bar{\gamma}>t L^{2}$ and $1>2 t \bar{\gamma}$. Then $0<1-t\left(2 \bar{\gamma}-t L^{2}\right)<1$ and $I-t V: H \rightarrow H$ is a contraction, where $I$ is the identity operator on $H$.

Lemma 8 ([21]) Let $H$ be a Hilbert space, and let $C$ be a nonempty closed convex subset of $H$. Let $P_{C}$ be the metric projection of $H$ onto $C$, and let $V$ be a $\bar{\gamma}$-strongly monotone and L-Lipschitzian continuous operator on $H$ with $\bar{\gamma}>0$ and $L>0$. Let $t>0$ satisfy $2 \bar{\gamma}>t L^{2}$ and $1>2 t \bar{\gamma}$, and let $z \in C$. Then the following are equivalent:

(1) $z=P_{C}(I-t V) z$;

(2) $\langle V z, y-z\rangle \geq 0, \forall y \in C$;

(3) $z=P_{C}(I-V) z$.

Such $z \in C$ always exists and is unique.

Lemma 9 ([21]) Let $H$ be a Hilbert space, and let $g: H \rightarrow H$ be a $k$-contraction with $0<$ $k<1$. Let $V$ be a $\bar{\gamma}$-strongly monotone and L-Lipschitzian continuous operator on $H$ with $\bar{\gamma}>0$ and $L>0$. Let a real number $\gamma$ satisfy $0<\gamma<\frac{\bar{\gamma}}{k}$. Then $V-\gamma g: H \rightarrow H$ is $a(\bar{\gamma}-\gamma k)-$ strongly monotone and $(L+\gamma k)$-Lipschitzian continuous mapping. Furthermore, let $C$ be a nonempty closed convex subset of $H$. Then $P_{C}(I-V+\gamma g)$ has a unique fixed point $z_{0}$ in $C$. This point $z_{0} \in C$ is also a unique solution of the variational inequality

$$
\left\langle(V-\gamma g) z_{0}, q-z_{0}\right\rangle \geq 0, \quad \forall q \in C
$$


Now, we prove the following strong convergence theorem of Halpern's type [6] for finding a common solution of a monotone inclusion problem for the sum of two monotone mappings, of a fixed point problem for generalized hybrid mappings and of an equilibrium problem for bifunctions in a Hilbert space.

Theorem 10 Let $H$ be a real Hilbert space, and let $C$ be a nonempty closed convex subset of $H$. Let $\alpha>0$, and let $A$ be an $\alpha$-inverse strongly-monotone mapping of $C$ into $H$. Let $B$ and $W$ be maximal monotone operators on $H$ such that the domains of $B$ and $W$ are included in $C$. Let $J_{\lambda}=(I+\lambda B)^{-1}$ and $T_{r}=(I+r W)^{-1}$ be resolvents of $B$ and $W$ for $\lambda>0$ and $r>0$, respectively. Let $S$ be a generalized hybrid mapping of $C$ into $H$. Let $0<k<1$, and let $g$ be a $k$-contraction of $H$ into itself. Let $V$ be a $\bar{\gamma}$-strongly monotone and L-Lipschitzian continuous operator with $\bar{\gamma}>0$ and $L>0$. Take $\mu, \gamma \in \mathbb{R}$ as follows:

$$
0<\mu<\frac{2 \bar{\gamma}}{L^{2}}, \quad 0<\gamma<\frac{\bar{\gamma}-\frac{L^{2} \mu}{2}}{k} .
$$

Suppose $F(S) \cap(A+B)^{-1} 0 \cap W^{-1} 0 \neq \emptyset$. Let $x_{1}=x \in H$, and let $\left\{x_{n}\right\} \subset H$ be a sequence generated by

$$
x_{n+1}=\beta_{n} x_{n}+\left(1-\beta_{n}\right)\left\{\alpha_{n} \gamma g\left(x_{n}\right)+\left(I-\alpha_{n} V\right) S J_{\lambda_{n}}\left(I-\lambda_{n} A\right) T_{r_{n}} x_{n}\right\}
$$

for all $n \in \mathbb{N}$, where $\left\{\alpha_{n}\right\} \subset(0,1),\left\{\beta_{n}\right\} \subset(0,1),\left\{\lambda_{n}\right\} \subset(0, \infty)$ and $\left\{r_{n}\right\} \subset(0, \infty)$ satisfy

$$
\begin{aligned}
& \lim _{n \rightarrow \infty} \alpha_{n}=0, \quad \sum_{n=1}^{\infty} \alpha_{n}=\infty, \quad 0<\liminf _{n \rightarrow \infty} \beta_{n} \leq \limsup _{n \rightarrow \infty} \beta_{n}<1, \\
& \liminf _{n \rightarrow \infty} r_{n}>0 \quad \text { and } \quad 0<a \leq \lambda_{n} \leq b<2 \alpha .
\end{aligned}
$$

Then $\left\{x_{n}\right\}$ converges strongly to $z_{0} \in F(S) \cap(A+B)^{-1} 0 \cap W^{-1} 0$, where $z_{0}$ is a unique fixed point in $F(S) \cap(A+B)^{-1} 0 \cap W^{-1} 0$ of $P_{F(S) \cap(A+B)^{-1} 0 \cap W^{-1} 0}(I-V+\gamma g)$.

Proof Let $z \in F(S) \cap(A+B)^{-1} 0 \cap W^{-1} 0$. We have that $z=S z, z=J_{\lambda_{n}}\left(I-\lambda_{n} A\right) z$ and $z=T_{r_{n}} z$. Putting $w_{n}=J_{\lambda_{n}}\left(I-\lambda_{n} A\right) T_{r_{n}} x_{n}$ and $u_{n}=T_{r_{n}} x_{n}$, we obtain that

$$
\begin{aligned}
\left\|S w_{n}-z\right\|^{2} & \leq\left\|w_{n}-z\right\|^{2} \\
& =\left\|J_{\lambda_{n}}\left(u_{n}-\lambda_{n} A u_{n}\right)-J_{\lambda_{n}}\left(z-\lambda_{n} A z\right)\right\|^{2} \\
& \leq\left\|u_{n}-\lambda_{n} A u_{n}-\left(z-\lambda_{n} A z\right)\right\|^{2} \\
& =\left\|u_{n}-z-\lambda_{n}\left(A u_{n}-A z\right)\right\|^{2} \\
& =\left\|u_{n}-z\right\|^{2}-2 \lambda_{n}\left\langle u_{n}-z, A u_{n}-A z\right\rangle+\lambda_{n}^{2}\left\|A u_{n}-A z\right\|^{2} \\
& \leq\left\|u_{n}-z\right\|^{2}-2 \lambda_{n} \alpha\left\|A u_{n}-A z\right\|^{2}+\lambda_{n}^{2}\left\|A u_{n}-A z\right\|^{2} \\
& \leq\left\|x_{n}-z\right\|^{2}+\lambda_{n}\left(\lambda_{n}-2 \alpha\right)\left\|A u_{n}-A z\right\|^{2} \\
& \leq\left\|x_{n}-z\right\|^{2} .
\end{aligned}
$$


Put $\tau=\bar{\gamma}-\frac{L^{2} \mu}{2}$. Using $\lim _{n \rightarrow \infty} \alpha_{n}=0$, we have that for any $x, y \in H$,

$$
\begin{aligned}
\| & \left(I-\alpha_{n} V\right) x-\left(I-\alpha_{n} V\right) y \|^{2} \\
& =\left\|x-y-\alpha_{n}(V x-V y)\right\|^{2} \\
& =\|x-y\|^{2}-2 \alpha_{n}\langle x-y, V x-V y\rangle+\alpha_{n}^{2}\|V x-V y\|^{2} \\
& \leq\|x-y\|^{2}-2 \alpha_{n} \bar{\gamma}\|x-y\|^{2}+\alpha_{n}^{2} L^{2}\|x-y\|^{2} \\
& =\left(1-2 \alpha_{n} \bar{\gamma}+\alpha_{n}^{2} L^{2}\right)\|x-y\|^{2} \\
& =\left(1-2 \alpha_{n} \tau-\alpha_{n} L^{2} \mu+\alpha_{n}^{2} L^{2}\right)\|x-y\|^{2} \\
& \leq\left(1-2 \alpha_{n} \tau-\alpha_{n}\left(L^{2} \mu-\alpha_{n} L^{2}\right)+\alpha_{n}^{2} \tau^{2}\right)\|x-y\|^{2} \\
& \leq\left(1-2 \alpha_{n} \tau+\alpha_{n}^{2} \tau^{2}\right)\|x-y\|^{2} \\
& =\left(1-\alpha_{n} \tau\right)^{2}\|x-y\|^{2} .
\end{aligned}
$$

Since $1-\alpha_{n} \tau>0$, we obtain that for any $x, y \in H$,

$$
\left\|\left(I-\alpha_{n} V\right) x-\left(I-\alpha_{n} V\right) y\right\| \leq\left(1-\alpha_{n} \tau\right)\|x-y\| .
$$

Putting $y_{n}=\alpha_{n} \gamma g\left(x_{n}\right)+\left(I-\alpha_{n} V\right) S J_{\lambda_{n}}\left(I-\lambda_{n} A\right) T_{r_{n}} x_{n}$, from $z=\alpha_{n} V z+z-\alpha_{n} V z$, (3.1) and (3.3) we have that

$$
\begin{aligned}
\left\|y_{n}-z\right\| & =\left\|\alpha_{n}\left(\gamma g\left(x_{n}\right)-V z\right)+\left(I-\alpha_{n} V\right) S w_{n}-\left(I-\alpha_{n} V\right) z\right\| \\
& \leq \alpha_{n} \gamma k\left\|x_{n}-z\right\|+\alpha_{n}\|\gamma g(z)-V z\|+\left(1-\alpha_{n} \tau\right)\left\|S w_{n}-z\right\| \\
& \leq\left\{1-\alpha_{n}(\tau-\gamma k)\right\}\left\|x_{n}-z\right\|+\alpha_{n}\|\gamma g(z)-V z\| .
\end{aligned}
$$

Using this, we get

$$
\begin{aligned}
\left\|x_{n+1}-z\right\|= & \left\|\beta_{n}\left(x_{n}-z\right)+\left(1-\beta_{n}\right)\left(y_{n}-z\right)\right\| \\
\leq & \beta_{n}\left\|x_{n}-z\right\|+\left(1-\beta_{n}\right)\left\|y_{n}-z\right\| \\
\leq & \beta_{n}\left\|x_{n}-z\right\| \\
& +\left(1-\beta_{n}\right)\left(\left\{1-\alpha_{n}(\tau-\gamma k)\right\}\left\|x_{n}-z\right\|+\alpha_{n}\|\gamma g(z)-V z\|\right) \\
= & \left\{1-\left(1-\beta_{n}\right) \alpha_{n}(\tau-\gamma k)\right\}\left\|x_{n}-z\right\| \\
& +\left(1-\beta_{n}\right) \alpha_{n}(\tau-\gamma k) \frac{\|\gamma g(z)-V z\|}{\tau-\gamma k} .
\end{aligned}
$$

Putting $K=\max \left\{\left\|x_{1}-z\right\|, \frac{\|\gamma g(z)-V z\|}{\tau-\gamma k}\right\}$, we have that $\left\|x_{n}-z\right\| \leq K$ for all $n \in \mathbb{N}$. Then $\left\{x_{n}\right\}$ is bounded. Furthermore, $\left\{u_{n}\right\},\left\{w_{n}\right\}$ and $\left\{y_{n}\right\}$ are bounded. Using Lemma 9, we can take a unique $z_{0} \in F(S) \cap(A+B)^{-1} 0 \cap W^{-1} 0$ such that

$$
z_{0}=P_{F(S) \cap(A+B)^{-1} 0 \cap W^{-1} 0}(I-V+\gamma g) z_{0} .
$$

From the definition of $\left\{x_{n}\right\}$, we have that

$$
x_{n+1}-x_{n}=\beta_{n} x_{n}+\left(1-\beta_{n}\right)\left\{\alpha_{n} \gamma g\left(x_{n}\right)+\left(I-\alpha_{n} V\right) S w_{n}\right\}-x_{n}
$$


and hence

$$
\begin{aligned}
x_{n+1}-x_{n}-\left(1-\beta_{n}\right) \alpha_{n} \gamma g\left(x_{n}\right) & =\beta_{n} x_{n}+\left(1-\beta_{n}\right)\left(I-\alpha_{n} V\right) S w_{n}-x_{n} \\
& =\left(1-\beta_{n}\right)\left\{\left(I-\alpha_{n} V\right) S w_{n}-x_{n}\right\} \\
& =\left(1-\beta_{n}\right)\left(S w_{n}-x_{n}-\alpha_{n} V S w_{n}\right) .
\end{aligned}
$$

Thus, we have that

$$
\begin{aligned}
& \left\langle x_{n+1}-x_{n}-\left(1-\beta_{n}\right) \alpha_{n} \gamma g\left(x_{n}\right), x_{n}-z_{0}\right\rangle \\
& \quad=\left\langle\left(1-\beta_{n}\right)\left(S w_{n}-x_{n}-\alpha_{n} V S w_{n}\right), x_{n}-z_{0}\right\rangle \\
& =-\left(1-\beta_{n}\right)\left\langle x_{n}-S w_{n}, x_{n}-z_{0}\right\rangle-\left(1-\beta_{n}\right) \alpha_{n}\left\langle V S w_{n}, x_{n}-z_{0}\right\rangle .
\end{aligned}
$$

From (2.3) and (3.1), we have that

$$
\begin{aligned}
2\left\langle x_{n}-S w_{n}, x_{n}-z_{0}\right\rangle & =\left\|x_{n}-z_{0}\right\|^{2}+\left\|S w_{n}-x_{n}\right\|^{2}-\left\|S w_{n}-z_{0}\right\|^{2} \\
& \geq\left\|x_{n}-z_{0}\right\|^{2}+\left\|S w_{n}-x_{n}\right\|^{2}-\left\|x_{n}-z_{0}\right\|^{2} \\
& =\left\|S w_{n}-x_{n}\right\|^{2} .
\end{aligned}
$$

From (3.4) and (3.5), we also have that

$$
\begin{aligned}
-2\left\langle x_{n}-x_{n+1}, x_{n}-z_{0}\right\rangle= & 2\left(1-\beta_{n}\right) \alpha_{n}\left\langle\gamma g\left(x_{n}\right), x_{n}-z_{0}\right\rangle \\
& -2\left(1-\beta_{n}\right)\left\langle x_{n}-S w_{n}, x_{n}-z_{0}\right\rangle-2\left(1-\beta_{n}\right) \alpha_{n}\left\langle V S w_{n}, x_{n}-z_{0}\right\rangle \\
\leq & 2\left(1-\beta_{n}\right) \alpha_{n}\left\langle\gamma g\left(x_{n}\right), x_{n}-z_{0}\right\rangle \\
& -\left(1-\beta_{n}\right)\left\|S w_{n}-x_{n}\right\|^{2}-2\left(1-\beta_{n}\right) \alpha_{n}\left\langle V S w_{n}, x_{n}-z_{0}\right\rangle .
\end{aligned}
$$

Furthermore, using (2.3) and (3.6), we have that

$$
\begin{aligned}
& \left\|x_{n+1}-z_{0}\right\|^{2}-\left\|x_{n}-x_{n+1}\right\|^{2}-\left\|x_{n}-z_{0}\right\|^{2} \\
& \leq 2\left(1-\beta_{n}\right) \alpha_{n}\left\langle\gamma g\left(x_{n}\right), x_{n}-z_{0}\right\rangle \\
& \quad-\left(1-\beta_{n}\right)\left\|S w_{n}-x_{n}\right\|^{2}-2\left(1-\beta_{n}\right) \alpha_{n}\left\langle V S w_{n}, x_{n}-z_{0}\right\rangle .
\end{aligned}
$$

Setting $\Gamma_{n}=\left\|x_{n}-z_{0}\right\|^{2}$, we have that

$$
\begin{aligned}
\Gamma_{n+1}- & \Gamma_{n}-\left\|x_{n}-x_{n+1}\right\|^{2} \\
\leq & 2\left(1-\beta_{n}\right) \alpha_{n}\left\langle\gamma g\left(x_{n}\right), x_{n}-z_{0}\right\rangle \\
& \quad\left(1-\beta_{n}\right)\left\|S w_{n}-x_{n}\right\|^{2}-2\left(1-\beta_{n}\right) \alpha_{n}\left\langle V S w_{n}, x_{n}-z_{0}\right\rangle .
\end{aligned}
$$

Noting that

$$
\begin{aligned}
\left\|x_{n+1}-x_{n}\right\| & =\left\|\left(1-\beta_{n}\right) \alpha_{n}\left(\gamma g\left(x_{n}\right)-V S w_{n}\right)+\left(1-\beta_{n}\right)\left(S w_{n}-x_{n}\right)\right\| \\
& \leq\left(1-\beta_{n}\right)\left(\left\|S w_{n}-x_{n}\right\|+\alpha_{n}\left\|\gamma g\left(x_{n}\right)-V S w_{n}\right\|\right),
\end{aligned}
$$


we have that

$$
\begin{aligned}
\left\|x_{n+1}-x_{n}\right\|^{2} \leq & \left(1-\beta_{n}\right)^{2}\left(\left\|S w_{n}-x_{n}\right\|+\alpha_{n}\left\|\gamma g\left(x_{n}\right)-V S w_{n}\right\|\right)^{2} \\
= & \left(1-\beta_{n}\right)^{2}\left\|S w_{n}-x_{n}\right\|^{2}+\left(1-\beta_{n}\right)^{2} 2 \alpha_{n}\left\|S w_{n}-x_{n}\right\|\left\|\gamma g\left(x_{n}\right)-V S w_{n}\right\| \\
& +\left(1-\beta_{n}\right)^{2} \alpha_{n}^{2}\left\|\gamma g\left(x_{n}\right)-V S w_{n}\right\|^{2} .
\end{aligned}
$$

Thus, we have from (3.7) and (3.9) that

$$
\begin{aligned}
\Gamma_{n+1}-\Gamma_{n} \leq & \left\|x_{n}-x_{n+1}\right\|^{2}+2\left(1-\beta_{n}\right) \alpha_{n}\left\langle\gamma g\left(x_{n}\right), x_{n}-z_{0}\right\rangle \\
& -\left(1-\beta_{n}\right)\left\|S w_{n}-x_{n}\right\|^{2}-2\left(1-\beta_{n}\right) \alpha_{n}\left\langle V S w_{n}, x_{n}-z_{0}\right\rangle \\
\leq & \left(1-\beta_{n}\right)^{2}\left\|S w_{n}-x_{n}\right\|^{2}+\left(1-\beta_{n}\right)^{2} 2 \alpha_{n}\left\|S w_{n}-x_{n}\right\|\left\|\gamma g\left(x_{n}\right)-V S w_{n}\right\| \\
& +\left(1-\beta_{n}\right)^{2} \alpha_{n}^{2}\left\|\gamma g\left(x_{n}\right)-V S w_{n}\right\|^{2}+2\left(1-\beta_{n}\right) \alpha_{n}\left\langle\gamma g\left(x_{n}\right), x_{n}-z_{0}\right\rangle \\
& -\left(1-\beta_{n}\right)\left\|S w_{n}-x_{n}\right\|^{2}-2\left(1-\beta_{n}\right) \alpha_{n}\left\langle V S w_{n}, x_{n}-z_{0}\right\rangle
\end{aligned}
$$

and hence

$$
\begin{aligned}
\Gamma_{n+1} & -\Gamma_{n}+\beta_{n}\left(1-\beta_{n}\right)\left\|S w_{n}-x_{n}\right\|^{2} \\
\leq & \left(1-\beta_{n}\right)^{2} 2 \alpha_{n}\left\|S w_{n}-x_{n}\right\|\left\|\gamma g\left(x_{n}\right)-V S w_{n}\right\| \\
& +\left(1-\beta_{n}\right)^{2} \alpha_{n}^{2}\left\|\gamma g\left(x_{n}\right)-V S w_{n}\right\|^{2}+2\left(1-\beta_{n}\right) \alpha_{n}\left\langle\gamma g\left(x_{n}\right), x_{n}-z_{0}\right\rangle \\
& -2\left(1-\beta_{n}\right) \alpha_{n}\left\langle V S w_{n}, x_{n}-z_{0}\right\rangle .
\end{aligned}
$$

We divide the proof into two cases.

Case 1: Suppose that $\Gamma_{n+1} \leq \Gamma_{n}$ for all $n \in \mathbb{N}$. In this case, $\lim _{n \rightarrow \infty} \Gamma_{n}$ exists and then $\lim _{n \rightarrow \infty}\left(\Gamma_{n+1}-\Gamma_{n}\right)=0$. Using $0<\liminf _{n \rightarrow \infty} \beta_{n} \leq \limsup _{n \rightarrow \infty} \beta_{n}<1$ and $\lim _{n \rightarrow \infty} \alpha_{n}=0$, we have from (3.10) that

$$
\lim _{n \rightarrow \infty}\left\|S w_{n}-x_{n}\right\|=0
$$

Using (3.8), we also have that

$$
\lim _{n \rightarrow \infty}\left\|x_{n+1}-x_{n}\right\|=0
$$

Since $x_{n+1}-x_{n}=\left(1-\beta_{n}\right)\left(y_{n}-x_{n}\right)$, we have from (3.12) that

$$
\lim _{n \rightarrow \infty}\left\|y_{n}-x_{n}\right\|=0
$$

We also have from (2.6) that

$$
\begin{aligned}
2\left\|u_{n}-z_{0}\right\|^{2} & =2\left\|T_{r_{n}} x_{n}-T_{r_{n}} z_{0}\right\|^{2} \\
& \leq 2\left\langle x_{n}-z_{0}, u_{n}-z_{0}\right\rangle \\
& =\left\|x_{n}-z_{0}\right\|^{2}+\left\|u_{n}-z_{0}\right\|^{2}-\left\|u_{n}-x_{n}\right\|^{2}
\end{aligned}
$$


and hence

$$
\left\|u_{n}-z_{0}\right\|^{2} \leq\left\|x_{n}-z_{0}\right\|^{2}-\left\|u_{n}-x_{n}\right\|^{2} .
$$

From (3.1) we have that

$$
\left\|S w_{n}-z_{0}\right\|^{2} \leq\left\|u_{n}-z_{0}\right\|^{2} \leq\left\|x_{n}-z_{0}\right\|^{2}-\left\|u_{n}-x_{n}\right\|^{2}
$$

and hence

$$
\left\|u_{n}-x_{n}\right\|^{2} \leq\left\|x_{n}-z_{0}\right\|^{2}-\left\|S w_{n}-z_{0}\right\|^{2} \leq M\left\|S w_{n}-x_{n}\right\|^{2},
$$

where $M=\sup \left\{\left\|x_{n}-z_{0}\right\|+\left\|S w_{n}-z_{0}\right\|: n \in \mathbb{N}\right\}$. Thus, from (3.11) we have that

$$
\lim _{n \rightarrow \infty}\left\|u_{n}-x_{n}\right\|=0
$$

We show $\lim _{n \rightarrow \infty}\left\|S w_{n}-w_{n}\right\|=0$. Since $\|\cdot\|^{2}$ is a convex function, we have that

$$
\left\|x_{n+1}-z_{0}\right\|^{2} \leq \beta_{n}\left\|x_{n}-z_{0}\right\|^{2}+\left(1-\beta_{n}\right)\left\|y_{n}-z_{0}\right\|^{2} .
$$

From $z_{0}=\alpha_{n} V z_{0}+z_{0}-\alpha_{n} V z_{0}$ and (2.1), we also have that

$$
\begin{aligned}
\left\|y_{n}-z_{0}\right\|^{2}= & \left\|\alpha_{n}\left(\gamma g\left(x_{n}\right)-V z_{0}\right)+\left(I-\alpha_{n} V\right) S w_{n}-\left(I-\alpha_{n} V\right) z_{0}\right\|^{2} \\
\leq & \left(1-\alpha_{n} \tau\right)^{2}\left\|S w_{n}-z_{0}\right\|^{2}+2 \alpha_{n}\left\langle\gamma g\left(x_{n}\right)-V z_{0}, y_{n}-z_{0}\right\rangle \\
\leq & \left(1-\alpha_{n} \tau\right)^{2}\left\|w_{n}-z_{0}\right\|^{2}+2 \alpha_{n}\left\langle\gamma g\left(x_{n}\right)-V z_{0}, y_{n}-z_{0}\right\rangle \\
\leq & \left\|w_{n}-z_{0}\right\|^{2}+2 \alpha_{n}\left\langle\gamma g\left(x_{n}\right)-V z_{0}, y_{n}-z_{0}\right\rangle \\
\leq & \left\|x_{n}-z\right\|^{2}+\lambda_{n}\left(\lambda_{n}-2 \alpha\right)\left\|A u_{n}-A z\right\|^{2} \\
& +2 \alpha_{n}\left\langle\gamma g\left(x_{n}\right)-V z_{0}, y_{n}-z_{0}\right\rangle .
\end{aligned}
$$

Using (3.16) and (3.17), we have that

$$
\begin{aligned}
\left\|x_{n+1}-z_{0}\right\|^{2} \leq & \beta_{n}\left\|x_{n}-z_{0}\right\|^{2}+\left(1-\beta_{n}\right)\left\|x_{n}-z_{0}\right\|^{2} \\
& +\left(1-\beta_{n}\right)\left(\lambda_{n}\left(\lambda_{n}-2 \alpha\right)\left\|A u_{n}-A z_{0}\right\|^{2}+2 \alpha_{n}\left\langle\gamma g\left(x_{n}\right)-V z_{0}, y_{n}-z_{0}\right\rangle\right) \\
= & \left\|x_{n}-z_{0}\right\|^{2}+\left(1-\beta_{n}\right)\left(\lambda_{n}\left(\lambda_{n}-2 \alpha\right)\left\|A u_{n}-A z_{0}\right\|^{2}\right. \\
& \left.+2 \alpha_{n}\left\langle\gamma g\left(x_{n}\right)-V z_{0}, y_{n}-z_{0}\right\rangle\right) .
\end{aligned}
$$

Thus, we have that

$$
\begin{aligned}
& \left(1-\beta_{n}\right) \lambda_{n}\left(2 \alpha-\lambda_{n}\right)\left\|A u_{n}-A z\right\|^{2} \\
& \quad \leq\left\|x_{n}-z\right\|^{2}-\left\|x_{n+1}-z\right\|^{2}+\left(1-\beta_{n}\right) 2 \alpha_{n}\left\langle\gamma g\left(x_{n}\right)-V z_{0}, y_{n}-z_{0}\right\rangle .
\end{aligned}
$$

Then we have that

$$
\lim _{n \rightarrow \infty}\left\|A u_{n}-A z_{0}\right\|=0
$$


Since $J_{\lambda_{n}}$ is firmly nonexpansive, we have that

$$
\begin{aligned}
2\left\|w_{n}-z_{0}\right\|^{2}= & 2\left\|J_{\lambda_{n}}\left(u_{n}-\lambda_{n} A u_{n}\right)-J_{\lambda_{n}}\left(z_{0}-\lambda_{n} A z_{0}\right)\right\|^{2} \\
\leq & 2\left\langle u_{n}-\lambda_{n} A u_{n}-\left(z_{0}-\lambda_{n} A z_{0}\right), w_{n}-z_{0}\right\rangle \\
= & \left\|u_{n}-\lambda_{n} A u_{n}-\left(z_{0}-\lambda_{n} A z_{0}\right)\right\|^{2}+\left\|w_{n}-z_{0}\right\|^{2} \\
& -\left\|u_{n}-\lambda_{n} A u_{n}-\left(z_{0}-\lambda_{n} A z_{0}\right)-\left(w_{n}-z_{0}\right)\right\|^{2} \\
\leq & \left\|u_{n}-z_{0}\right\|^{2}+\left\|w_{n}-z_{0}\right\|^{2} \\
& \quad-\left\|u_{n}-w_{n}-\lambda_{n}\left(A u_{n}-A z_{0}\right)\right\|^{2} \\
\leq & \left\|x_{n}-z_{0}\right\|^{2}+\left\|w_{n}-z_{0}\right\|^{2}-\left\|u_{n}-w_{n}\right\|^{2} \\
& +2 \lambda_{n}\left\langle u_{n}-w_{n}, A u_{n}-A z_{0}\right\rangle-\lambda_{n}^{2}\left\|A u_{n}-A z_{0}\right\|^{2} .
\end{aligned}
$$

Thus, we get

$$
\begin{aligned}
\left\|w_{n}-z_{0}\right\|^{2} \leq & \left\|x_{n}-z_{0}\right\|^{2}-\left\|u_{n}-w_{n}\right\|^{2} \\
& +2 \lambda_{n}\left\langle u_{n}-w_{n}, A u_{n}-A z_{0}\right\rangle-\lambda_{n}^{2}\left\|A u_{n}-A z_{0}\right\|^{2} .
\end{aligned}
$$

Using (3.17), we obtain

$$
\begin{aligned}
\left\|x_{n+1}-z_{0}\right\|^{2} \leq & \beta_{n}\left\|x_{n}-z_{0}\right\|^{2}+\left(1-\beta_{n}\right)\left\|y_{n}-z_{0}\right\|^{2} \\
\leq & \beta_{n}\left\|x_{n}-z_{0}\right\|^{2}+\left(1-\beta_{n}\right)\left(\left\|w_{n}-z_{0}\right\|^{2}+2 \alpha_{n}\left\langle\gamma g\left(x_{n}\right)-V z_{0}, y_{n}-z_{0}\right\rangle\right) \\
\leq & \beta_{n}\left\|x_{n}-z_{0}\right\|^{2}+\left(1-\beta_{n}\right)\left\|x_{n}-z_{0}\right\|^{2} \\
& -\left(1-\beta_{n}\right)\left\|u_{n}-w_{n}\right\|^{2}+\left(1-\beta_{n}\right) 2 \lambda_{n}\left\langle u_{n}-w_{n}, A u_{n}-A z_{0}\right\rangle \\
& -\left(1-\beta_{n}\right) \lambda_{n}^{2}\left\|A u_{n}-A z_{0}\right\|^{2}+\left(1-\beta_{n}\right) 2 \alpha_{n}\left\langle\gamma g\left(x_{n}\right)-V z_{0}, y_{n}-z_{0}\right\rangle \\
= & \left\|x_{n}-z_{0}\right\|^{2}-\left(1-\beta_{n}\right)\left\|u_{n}-w_{n}\right\|^{2} \\
& +\left(1-\beta_{n}\right) 2 \lambda_{n}\left\langle u_{n}-w_{n}, A u_{n}-A z_{0}\right\rangle-\left(1-\beta_{n}\right) \lambda_{n}^{2}\left\|A u_{n}-A z_{0}\right\|^{2} \\
& +\left(1-\beta_{n}\right) 2 \alpha_{n}\left\langle\gamma g\left(x_{n}\right)-V z_{0}, y_{n}-z_{0}\right\rangle,
\end{aligned}
$$

from which it follows that

$$
\begin{aligned}
\left(1-\beta_{n}\right)\left\|x_{n}-w_{n}\right\|^{2} \leq & \left\|x_{n}-z_{0}\right\|^{2} \\
& -\left\|x_{n+1}-z_{0}\right\|^{2}+2 \lambda_{n}\left\langle u_{n}-w_{n}, A u_{n}-A z_{0}\right\rangle \\
& -\lambda_{n}^{2}\left\|A u_{n}-A z_{0}\right\|^{2}+2 \alpha_{n}\left\langle\gamma g\left(x_{n}\right)-V z_{0}, y_{n}-z_{0}\right\rangle .
\end{aligned}
$$

Then we have

$$
\lim _{n \rightarrow \infty}\left\|u_{n}-w_{n}\right\|=0
$$

From (3.22) and (3.15), we have that

$$
\lim _{n \rightarrow \infty}\left\|x_{n}-w_{n}\right\|=0
$$


Since $\left\|S w_{n}-w_{n}\right\| \leq\left\|S w_{n}-x_{n}\right\|+\left\|x_{n}-w_{n}\right\|$, we have that

$$
\lim _{n \rightarrow \infty}\left\|S w_{n}-w_{n}\right\|=0
$$

Take $\lambda_{0} \in \mathbb{R}$ with $0<a \leq \lambda_{0} \leq b<2 \alpha$ arbitrarily. Put $s_{n}=\left(I-\lambda_{n} A\right) u_{n}$. Using $u_{n}=T_{r_{n}} x_{n}$ and $w_{n}=J_{\lambda_{n}}\left(I-\lambda_{n} A\right) u_{n}$, we have from Lemma 4 that

$$
\begin{aligned}
\left\|J_{\lambda_{0}}\left(I-\lambda_{0} A\right) u_{n}-w_{n}\right\|= & \left\|J_{\lambda_{0}}\left(I-\lambda_{0} A\right) u_{n}-J_{\lambda_{n}}\left(I-\lambda_{n} A\right) u_{n}\right\| \\
= & \| J_{\lambda_{0}}\left(I-\lambda_{0} A\right) u_{n}-J_{\lambda_{0}}\left(I-\lambda_{n} A\right) u_{n} \\
& +J_{\lambda_{0}}\left(I-\lambda_{n} A\right) u_{n}-J_{\lambda_{n}}\left(I-\lambda_{n} A\right) u_{n} \| \\
\leq & \left\|\left(I-\lambda_{0} A\right) u_{n}-\left(I-\lambda_{n} A\right) u_{n}\right\|+\left\|J_{\lambda_{0}} s_{n}-J_{\lambda_{n}} s_{n}\right\| \\
\leq & \left|\lambda_{0}-\lambda_{n}\right|\left\|A u_{n}\right\|+\frac{\left|\lambda_{0}-\lambda_{n}\right|}{\lambda_{0}}\left\|J_{\lambda_{0}} s_{n}-s_{n}\right\| .
\end{aligned}
$$

We also have from (3.25) that

$$
\left\|u_{n}-J_{\lambda_{0}}\left(I-\lambda_{0} A\right) u_{n}\right\| \leq\left\|u_{n}-w_{n}\right\|+\left\|w_{n}-J_{\lambda_{0}}\left(I-\lambda_{0} A\right) u_{n}\right\|
$$

We will use (3.25) and (3.26) later.

Let us show that $\limsup _{n \rightarrow \infty}\left\langle(V-\gamma g) z_{0}, x_{n}-z_{0}\right\rangle \geq 0$. Put

$$
A=\limsup _{n \rightarrow \infty}\left\langle(V-\gamma g) z_{0}, x_{n}-z_{0}\right\rangle
$$

Without loss of generality, we may assume that there exists a subsequence $\left\{x_{n_{i}}\right\}$ of $\left\{x_{n}\right\}$ such that $A=\lim _{i \rightarrow \infty}\left\langle(V-\gamma g) z_{0}, x_{n_{i}}-z_{0}\right\rangle$ and $\left\{x_{n_{i}}\right\}$ converges weakly to some point $w \in H$. From $\left\|x_{n}-w_{n}\right\| \rightarrow 0$ and $\left\|x_{n}-u_{n}\right\| \rightarrow 0$, we also have that $\left\{w_{n_{i}}\right\}$ and $\left\{u_{n_{i}}\right\}$ converge weakly to $w \in C$. On the other hand, from $\left\{\lambda_{n_{i}}\right\} \subset[a, b]$ there exists a subsequence $\left\{\lambda_{n_{i j}}\right\}$ of $\left\{\lambda_{n_{i}}\right\}$ such that $\lambda_{n_{i_{j}}} \rightarrow \lambda_{0}$ for some $\lambda_{0} \in[a, b]$. Without loss of generality, we assume that $w_{n_{i}} \rightarrow$ $w, u_{n_{i}} \rightarrow w$ and $\lambda_{n_{i}} \rightarrow \lambda_{0}$. From (3.24) we know $\lim _{n \rightarrow \infty}\left\|S w_{n}-w_{n}\right\|=0$. Thus, we have from Lemma 3 that $w=S w$. Since $W$ is a monotone operator and $\frac{x_{n_{i}}-u_{n_{i}}}{r_{n_{i}}} \in W u_{n_{i}}$, we have that for any $(u, v) \in W$,

$$
\left\langle u-u_{n_{i}}, v-\frac{x_{n_{i}}-u_{n_{i}}}{r_{n_{i}}}\right\rangle \geq 0 .
$$

Since $\liminf _{n \rightarrow \infty} r_{n}>0, u_{n_{i}} \rightarrow w$ and $x_{n_{i}}-u_{n_{i}} \rightarrow 0$, we have

$$
\langle u-w, v\rangle \geq 0 \text {. }
$$

Since $W$ is a maximal monotone operator, we have $0 \in W w$ and hence $w \in W^{-1} 0$. Since $\lambda_{n_{i}} \rightarrow \lambda_{0}$, we have from (3.25) that

$$
\left\|J_{\lambda_{0}}\left(I-\lambda_{0} A\right) u_{n_{i}}-w_{n_{i}}\right\| \rightarrow 0
$$

Furthermore, we have from (3.26) that

$$
\left\|u_{n_{i}}-J_{\lambda_{0}}\left(I-\lambda_{0} A\right) u_{n_{i}}\right\| \rightarrow 0
$$


Since $J_{\lambda_{0}}\left(I-\lambda_{0} A\right)$ is nonexpansive, we have that $w=J_{\lambda_{0}}\left(I-\lambda_{0} A\right) w$. This means that $0 \in$ $A w+B w$. Thus, we have

$$
w \in F(T) \cap(A+B)^{-1} 0 \cap W^{-1} 0 .
$$

Then we have

$$
A=\lim _{i \rightarrow \infty}\left\langle(V-\gamma g) z_{0}, x_{n_{i}}-z_{0}\right\rangle=\left\langle(V-\gamma g) z_{0}, w-z_{0}\right\rangle \geq 0 .
$$

Since $y_{n}-z_{0}=\alpha_{n}\left(\gamma g\left(x_{n}\right)-V z_{0}\right)+\left(I-\alpha_{n} V\right) S w_{n}-\left(I-\alpha_{n} V\right) z_{0}$, we have

$$
\left\|y_{n}-z_{0}\right\|^{2} \leq\left(1-\alpha_{n} \tau\right)^{2}\left\|S w_{n}-z_{0}\right\|^{2}+2 \alpha_{n}\left\langle\gamma g\left(x_{n}\right)-V z_{0}, y_{n}-z_{0}\right\rangle .
$$

Thus, we have

$$
\left\|y_{n}-z_{0}\right\|^{2} \leq\left(1-\alpha_{n} \tau\right)^{2}\left\|x_{n}-z_{0}\right\|^{2}+2 \alpha_{n}\left\langle\gamma g\left(x_{n}\right)-V z_{0}, y_{n}-z_{0}\right\rangle .
$$

Consequently, we have that

$$
\begin{aligned}
\left\|x_{n+1}-z_{0}\right\|^{2} \leq & \beta_{n}\left\|x_{n}-z_{0}\right\|^{2}+\left(1-\beta_{n}\right)\left\|y_{n}-z_{0}\right\|^{2} \\
\leq & \beta_{n}\left\|x_{n}-z_{0}\right\|^{2} \\
& +\left(1-\beta_{n}\right)\left(\left(1-\alpha_{n} \tau\right)^{2}\left\|x_{n}-z_{0}\right\|^{2}+2 \alpha_{n}\left\langle\gamma g\left(x_{n}\right)-V z_{0}, y_{n}-z_{0}\right\rangle\right) \\
= & \left(\beta_{n}+\left(1-\beta_{n}\right)\left(1-\alpha_{n} \tau\right)^{2}\right)\left\|x_{n}-z_{0}\right\|^{2} \\
& +2\left(1-\beta_{n}\right) \alpha_{n}\left\langle\gamma g\left(x_{n}\right)-V z_{0}, y_{n}-z_{0}\right\rangle \\
\leq & \left(1-\left(1-\beta_{n}\right)\left(2 \alpha_{n} \tau-\left(\alpha_{n} \tau\right)^{2}\right)\right)\left\|x_{n}-z_{0}\right\|^{2} \\
& +2\left(1-\beta_{n}\right) \alpha_{n} \gamma k\left\|x_{n}-z_{0}\right\|^{2}+2\left(1-\beta_{n}\right) \alpha_{n}\left\langle\gamma g\left(z_{0}\right)-V z_{0}, y_{n}-z_{0}\right\rangle \\
= & \left(1-2\left(1-\beta_{n}\right) \alpha_{n}(\tau-\gamma k)\right)\left\|x_{n}-z_{0}\right\|^{2} \\
& +\left(1-\beta_{n}\right)\left(\alpha_{n} \tau\right)^{2}\left\|x_{n}-z_{0}\right\|^{2}+2\left(1-\beta_{n}\right) \alpha_{n}\left\langle\gamma g\left(z_{0}\right)-V z_{0}, y_{n}-z_{0}\right\rangle \\
= & \left(1-2\left(1-\beta_{n}\right) \alpha_{n}(\tau-\gamma k)\right)\left\|x_{n}-z_{0}\right\|^{2} \\
& +2\left(1-\beta_{n}\right) \alpha_{n}(\tau-\gamma k)\left(\frac{\alpha_{n} \tau^{2}\left\|x_{n}-z_{0}\right\|^{2}}{2(\tau-\gamma k)}+\frac{\left\langle\gamma g\left(z_{0}\right)-V z_{0}, y_{n}-z_{0}\right\rangle}{\tau-\gamma k}\right) .
\end{aligned}
$$

By (3.27) and Lemma 5 , we obtain that $x_{n} \rightarrow z_{0}$, where

$$
z_{0}=P_{F(S) \cap(A+B)^{-1} 0 \cap W^{-1} 0}(I-V+\gamma g) z_{0} .
$$

Case 2: Suppose that there exists a subsequence $\left\{\Gamma_{n_{i}}\right\} \subset\left\{\Gamma_{n}\right\}$ such that $\Gamma_{n_{i}}<\Gamma_{n_{i}+1}$ for all $i \in \mathbb{N}$. In this case, we define $\tau: \mathbb{N} \rightarrow \mathbb{N}$ by

$$
\tau(n)=\max \left\{k \leq n: \Gamma_{k}<\Gamma_{k+1}\right\} .
$$


Then we have from Lemma 6 that $\Gamma_{\tau(n)}<\Gamma_{\tau(n)+1}$. Thus, we have from (3.10) that for all $n \in \mathbb{N}$

$$
\begin{aligned}
& \beta_{\tau(n)}\left(1-\beta_{\tau(n)}\right)\left\|S w_{\tau(n)}-x_{\tau(n)}\right\|^{2} \\
& \leq\left(1-\beta_{\tau(n)}\right)^{2} 2 \alpha_{\tau(n)}\left\|S w_{\tau(n)}-x_{\tau(n)}\right\|\left\|\gamma g\left(x_{\tau(n)}\right)-V S w_{\tau(n)}\right\| \\
& \quad+\left(1-\beta_{\tau(n)}\right)^{2} \alpha_{\tau(n)}^{2}\left\|\gamma g\left(x_{\tau(n)}\right)-V S w_{\tau(n)}\right\|^{2} \\
& \quad+2\left(1-\beta_{\tau(n)}\right) \alpha_{\tau(n)}\left\langle\gamma g\left(x_{\tau(n)}\right), x_{\tau(n)}-z_{0}\right\rangle \\
& \quad-2\left(1-\beta_{\tau(n)}\right) \alpha_{\tau(n)}\left\langle V S w_{\tau(n)}, x_{\tau(n)}-z_{0}\right\rangle .
\end{aligned}
$$

Using $\lim _{n \rightarrow \infty} \alpha_{n}=0$ and $0<\liminf _{n \rightarrow \infty} \beta_{n} \leq \lim \sup _{n \rightarrow \infty} \beta_{n}<1$, we have from (3.28) and Lemma 6 that

$$
\lim _{n \rightarrow \infty}\left\|S w_{\tau}(n)-x_{\tau}(n)\right\|=0
$$

As in the proof of Case 1, we also have that

$$
\lim _{n \rightarrow \infty}\left\|x_{\tau(n)+1}-x_{\tau(n)}\right\|=0
$$

and

$$
\lim _{n \rightarrow \infty}\left\|y_{\tau(n)}-x_{\tau(n)}\right\|=0
$$

Furthermore, we have that $\lim _{n \rightarrow \infty}\left\|u_{\tau(n)}-x_{\tau(n)}\right\|=0, \lim _{n \rightarrow \infty}\left\|A u_{\tau(n)}-A z_{0}\right\|=0$, $\lim _{n \rightarrow \infty}\left\|u_{\tau(n)}-w_{\tau(n)}\right\|=0$ and $\lim _{n \rightarrow \infty}\left\|x_{\tau(n)}-w_{\tau(n)}\right\|=0$. From these we have that $\lim _{n \rightarrow \infty}\left\|S w_{\tau(n)}-w_{\tau(n)}\right\|=0$. As in the proof of Case 1, we can show that

$$
\limsup _{n \rightarrow \infty}\left\{(V-\gamma g) z_{0}, x_{\tau(n)}-z_{0}\right\rangle \geq 0
$$

We also have that

$$
\left\|y_{\tau(n)}-z_{0}\right\|^{2} \leq\left(1-\alpha_{\tau(n)} \tau\right)^{2}\left\|x_{\tau(n)}-z_{0}\right\|^{2}+2 \alpha_{\tau(n)}\left\langle\gamma g\left(x_{\tau(n)}\right)-V z_{0}, y_{\tau(n)}-z_{0}\right\rangle
$$

and hence

$$
\begin{aligned}
\left\|x_{\tau(n)+1}-z_{0}\right\|^{2} \leq & \left(1-2\left(1-\beta_{\tau(n)}\right) \alpha_{\tau(n)}(\tau-\gamma k)\right)\left\|x_{\tau(n)}-z_{0}\right\|^{2} \\
& +\left(1-\beta_{\tau(n)}\right)\left(\alpha_{\tau(n)} \tau\right)^{2}\left\|x_{\tau(n)}-z_{0}\right\|^{2} \\
& +2\left(1-\beta_{\tau(n)}\right) \alpha_{\tau(n)}\left\langle\gamma g\left(z_{0}\right)-V z_{0}, y_{\tau(n)}-z_{0}\right\rangle .
\end{aligned}
$$

From $\Gamma_{\tau(n)}<\Gamma_{\tau(n)+1}$, we have that

$$
\begin{aligned}
2(1- & \left.\beta_{\tau(n)}\right) \alpha_{\tau(n)}(\tau-\gamma k)\left\|x_{\tau(n)}-z_{0}\right\|^{2} \\
\leq & \left(1-\beta_{\tau(n)}\right)\left(\alpha_{\tau(n)} \tau\right)^{2}\left\|x_{\tau(n)}-z_{0}\right\|^{2} \\
& \left.\quad+2\left(1-\beta_{\tau(n)}\right) \alpha_{\tau(n)}\right)\left(\gamma g\left(z_{0}\right)-V z_{0}, y_{\tau(n)}-z_{0}\right) .
\end{aligned}
$$


Since $\left(1-\beta_{\tau(n)}\right) \alpha_{\tau(n)}>0$, we have that

$$
\begin{aligned}
& 2(\tau-\gamma k)\left\|x_{\tau(n)}-z_{0}\right\|^{2} \\
& \quad \leq \alpha_{\tau(n)} \tau^{2}\left\|x_{\tau(n)}-z_{0}\right\|^{2}+2\left\langle\gamma g\left(z_{0}\right)-V z_{0}, y_{\tau(n)}-z_{0}\right) .
\end{aligned}
$$

Thus, we have that

$$
\limsup _{n \rightarrow \infty} 2(\tau-\gamma k)\left\|x_{\tau(n)}-z_{0}\right\|^{2} \leq 0
$$

and hence $\left\|x_{\tau(n)}-z_{0}\right\| \rightarrow 0$ as $n \rightarrow \infty$. Since $x_{\tau(n)}-x_{\tau(n)+1} \rightarrow 0$, we have $\left\|x_{\tau(n)+1}-z_{0}\right\| \rightarrow 0$ as $n \rightarrow \infty$. Using Lemma 6 again, we obtain that

$$
\left\|x_{n}-z_{0}\right\| \leq\left\|x_{\tau(n)+1}-z_{0}\right\| \rightarrow 0
$$

as $n \rightarrow \infty$. This completes the proof.

\section{Applications}

In this section, using Theorem 10, we can obtain well-known and new strong convergence theorems in a Hilbert space. Let $H$ be a Hilbert space, and let $f$ be a proper lower semicontinuous convex function of $H$ into $(-\infty, \infty]$. Then the subdifferential $\partial f$ of $f$ is defined as follows:

$$
\partial f(x)=\{z \in H: f(x)+\langle z, y-x\rangle \leq f(y), \forall y \in H\}
$$

for all $x \in H$. From Rockafellar [22], we know that $\partial f$ is a maximal monotone operator. Let $C$ be a nonempty closed convex subset of $H$, and let $i_{C}$ be the indicator function of $C$, i.e.,

$$
i_{C}(x)= \begin{cases}0, & x \in C, \\ \infty, & x \notin C .\end{cases}
$$

Then, $i_{C}$ is a proper lower semicontinuous convex function on $H$. So, we can define the resolvent $J_{\lambda}$ of $\partial i_{C}$ for $\lambda>0$, i.e.,

$$
J_{\lambda} x=\left(I+\lambda \partial i_{C}\right)^{-1} x
$$

for all $x \in H$. We know that $J_{\lambda} x=P_{C} x$ for all $x \in H$ and $\lambda>0$; see [11].

Theorem 11 Let $H$ be a real Hilbert space, and let $C$ be a nonempty closed convex subset of $H$. Let $S$ be a generalized hybrid mapping of $C$ into $C$. Suppose $F(S) \neq \emptyset$. Let $u, x_{1} \in C$, and let $\left\{x_{n}\right\} \subset C$ be a sequence generated by

$$
x_{n+1}=\beta_{n} x_{n}+\left(1-\beta_{n}\right)\left\{\alpha_{n} u+\left(1-\alpha_{n}\right) S x_{n}\right\}
$$

for all $n \in \mathbb{N}$, where $\left\{\beta_{n}\right\} \subset(0,1)$ and $\left\{\alpha_{n}\right\} \subset(0,1)$ satisfy

$$
\lim _{n \rightarrow \infty} \alpha_{n}=0, \quad \sum_{n=1}^{\infty} \alpha_{n}=\infty
$$


and

$$
0<\liminf _{n \rightarrow \infty} \beta_{n} \leq \limsup _{n \rightarrow \infty} \beta_{n}<1
$$

Then the sequence $\left\{x_{n}\right\}$ converges strongly to $z_{0} \in F(S)$, where $z_{0}=P_{F(S)} u$.

Proof Put $A=0, B=W=\partial i_{C}$ and $\lambda_{n}=r_{n}=1$ for all $n \in \mathbb{N}$ in Theorem 10. Then we have $J_{\lambda_{n}}=T_{r_{n}}=P_{C}$ for all $n \in \mathbb{N}$. Furthermore, put $g(x)=u$ and $V(x)=x$ for all $x \in H$. Then we can take $\bar{\gamma}=L=1$. Thus, we can take $\mu=1$. On the other hand, since $\|g(x)-g(y)\|=0 \leq$ $\frac{1}{3}\|x-y\|$ for all $x, y \in H$, we can take $k=\frac{1}{3}$. So, we can take $\gamma=1$. Then for $u, x_{1} \in C$, we get that

$$
x_{n+1}=\beta_{n} x_{n}+\left(1-\beta_{n}\right)\left\{\alpha_{n} u+\left(I-\alpha_{n}\right) S x_{n}\right\}
$$

for all $n \in \mathbb{N}$. So, we have $\left\{x_{n}\right\} \subset C$. We also have

$$
z_{0}=P_{F(S) \cap C}(I-V+\gamma g) z_{0}=P_{F(S)}\left(z_{0}-z_{0}+1 \cdot u\right)=P_{F(S)} u
$$

Thus, we obtain the desired result by Theorem 10 .

Theorem 11 solves the problem posed by Kurokawa and Takahashi [9]. The following result is a strong convergence theorem of Halpern's type [6] for finding a common solution of a monotone inclusion problem for the sum of two monotone mappings, of a fixed point problem for nonexpansive mappings and of an equilibrium problem for bifunctions in a Hilbert space.

Theorem 12 Let $H$ be a real Hilbert space, and let $C$ be a nonempty closed convex subset of $H$. Let $\alpha>0$, and let $A$ be an $\alpha$-inverse strongly-monotone mapping of $C$ into $H$. Let $B$ and $W$ be maximal monotone operators on $H$ such that the domains of $B$ and $W$ are included in C. Let $J_{\lambda}=(I+\lambda B)^{-1}$ and $T_{r}=(I+r W)^{-1}$ be resolvents of $B$ and $W$ for $\lambda>0$ and $r>0$, respectively. Let $S$ be a nonexpansive mapping of $C$ into $H$. Let $0<k<1$, and let $g$ be a $k$-contraction of $H$ into itself. Let $V$ be a $\bar{\gamma}$-strongly monotone and L-Lipschitzian continuous operator with $\bar{\gamma}>0$ and $L>0$. Take $\mu, \gamma \in \mathbb{R}$ as follows:

$$
0<\mu<\frac{2 \bar{\gamma}}{L^{2}}, \quad 0<\gamma<\frac{\bar{\gamma}-\frac{L^{2} \mu}{2}}{k} .
$$

Suppose $F(S) \cap(A+B)^{-1} 0 \cap W^{-1} 0 \neq \emptyset$. Let $x_{1}=x \in H$, and let $\left\{x_{n}\right\} \subset H$ be a sequence generated by

$$
x_{n+1}=\beta_{n} x_{n}+\left(1-\beta_{n}\right)\left\{\alpha_{n} \gamma g\left(x_{n}\right)+\left(I-\alpha_{n} V\right) S J_{\lambda_{n}}\left(I-\lambda_{n} A\right) T_{r_{n}} x_{n}\right\}
$$

for all $n \in \mathbb{N}$, where $\left\{\alpha_{n}\right\} \subset(0,1),\left\{\beta_{n}\right\} \subset(0,1),\left\{\lambda_{n}\right\} \subset(0, \infty)$ and $\left\{r_{n}\right\} \subset(0, \infty)$ satisfy

$$
\begin{aligned}
& \lim _{n \rightarrow \infty} \alpha_{n}=0, \quad \sum_{n=1}^{\infty} \alpha_{n}=\infty, \quad 0<\liminf _{n \rightarrow \infty} \beta_{n} \leq \limsup _{n \rightarrow \infty} \beta_{n}<1, \\
& \liminf _{n \rightarrow \infty} r_{n}>0 \quad \text { and } \quad 0<a \leq \lambda_{n} \leq b<2 \alpha .
\end{aligned}
$$


Then the sequence $\left\{x_{n}\right\}$ converges strongly to $z_{0} \in F(S) \cap(A+B)^{-1} 0 \cap W^{-1} 0$, where $z_{0}=$ $P_{F(S) \cap(A+B)^{-1} 0 \cap W^{-1} 0}(I-V+\gamma g) z_{0}$.

Proof We know that a nonexpansive mapping $T$ of $C$ into $H$ is a $(1,0)$-generalized hybrid mapping. So, we obtain the desired result by Theorem 10 .

Let $f: C \times C \rightarrow \mathbb{R}$ be a bifunction. The equilibrium problem (with respect to $C$ ) is to find $\hat{x} \in C$ such that

$$
f(\hat{x}, y) \geq 0, \quad \forall y \in C .
$$

The set of such solutions $\hat{x}$ is denoted by $E P(f)$, i.e.,

$$
E P(f)=\{\hat{x} \in C: f(\hat{x}, y) \geq 0, \forall y \in C\} .
$$

For solving the equilibrium problem, let us assume that the bifunction $f: C \times C \rightarrow \mathbb{R}$ satisfies the following conditions:

(A1) $f(x, x)=0$ for all $x \in C$;

(A2) $f$ is monotone, i.e., $f(x, y)+f(y, x) \leq 0$ for all $x, y \in C$;

(A3) for all $x, y, z \in C$,

$$
\limsup _{t \downarrow 0} f(t z+(1-t) x, y) \leq f(x, y)
$$

(A4) for all $x \in C, f(x, \cdot)$ is convex and lower semicontinuous.

The following lemmas were given in Combettes and Hirstoaga [23] and Takahashi, Takahashi and Toyoda [16]; see also [24, 25].

Lemma 13 ([23]) Let $H$ be a real Hilbert space, and let $C$ be a nonempty closed convex subset of $H$. Assume that $f: C \times C \rightarrow \mathbb{R}$ satisfies (A1)-(A4). For $r>0$ and $x \in H$, define a mapping $T_{r}: H \rightarrow C$ as follows:

$$
T_{r} x=\left\{z \in C: f(z, y)+\frac{1}{r}\langle y-z, z-x\rangle \geq 0, \forall y \in C\right\}
$$

for all $x \in H$. Then the following hold:

(1) $T_{r}$ is single-valued;

(2) $T_{r}$ is a firmly nonexpansive mapping, i.e., for all $x, y \in H$,

$$
\left\|T_{r} x-T_{r} y\right\|^{2} \leq\left\langle T_{r} x-T_{r} y, x-y\right\rangle ;
$$

(3) $F\left(T_{r}\right)=E P(f)$;

(4) $E P(f)$ is closed and convex.

We call such $T_{r}$ the resolvent of $f$ for $r>0$.

Lemma 14 ([16]) Let $H$ be a Hilbert space, and let $C$ be a nonempty closed convex subset of $H$. Let $f: C \times C \rightarrow \mathbb{R}$ satisfy (A1)-(A4). Let $A_{f}$ be a set-valued mapping of $H$ into itself 
defined by

$$
A_{f} x= \begin{cases}\{z \in H: f(x, y) \geq\langle y-x, z\rangle, \forall y \in C\}, & \forall x \in C, \\ \emptyset, & \forall x \notin C .\end{cases}
$$

Then $E P(f)=A_{f}^{-1} 0$ and $A_{f}$ is a maximal monotone operator with $D\left(A_{f}\right) \subset C$. Furthermore, for any $x \in H$ and $r>0$, the resolvent $T_{r}$ of $f$ coincides with the resolvent of $A_{f}$, i.e.,

$$
T_{r} x=\left(I+r A_{f}\right)^{-1} x
$$

Using Lemmas 13,14 and Theorem 10, we also obtain the following result for generalized hybrid mappings of $C$ into $H$ with equilibrium problem in a Hilbert space; see also [2628].

Theorem 15 Let $H$ be a real Hilbert space, and let $C$ be a nonempty closed convex subset of $H$. Let $S$ be a generalised hybrid mapping of $C$ into $H$. Let $f$ be a bifunction of $C \times C$ into $\mathbb{R}$ satisfying (A1)-(A4). Let $0<k<1$, and let $g$ be a $k$-contraction of $H$ into itself. Let $V$ be $a \bar{\gamma}$-strongly monotone and L-Lipschitzian continuous operator of $H$ into itself with $\bar{\gamma}>0$ and $L>0$. Take $\mu, \gamma \in \mathbb{R}$ as follows:

$$
0<\mu<\frac{2 \bar{\gamma}}{L^{2}}, \quad 0<\gamma<\frac{\bar{\gamma}-\frac{L^{2} \mu}{2}}{k} .
$$

Suppose that $F(S) \cap E P(f) \neq \emptyset$. Let $x_{1}=x \in H$, and let $\left\{x_{n}\right\} \subset H$ be a sequence generated by

$$
\begin{aligned}
& f\left(u_{n}, y\right)+\frac{1}{r_{n}}\left\langle y-u_{n}, u_{n}-x_{n}\right\rangle \geq 0, \quad \forall y \in C, \\
& x_{n+1}=\beta_{n} x_{n}+\left(1-\beta_{n}\right)\left\{\alpha_{n} \gamma g\left(x_{n}\right)+\left(I-\alpha_{n} V\right) S u_{n}\right\}
\end{aligned}
$$

for all $n \in \mathbb{N}$, where $\left\{\beta_{n}\right\} \subset(0,1),\left\{\alpha_{n}\right\} \subset(0,1)$ and $\left\{r_{n}\right\} \subset(0, \infty)$ satisfy

$$
\begin{aligned}
& \lim _{n \rightarrow \infty} \alpha_{n}=0, \quad \sum_{n=1}^{\infty} \alpha_{n}=\infty, \quad \liminf _{n \rightarrow \infty} r_{n}>0, \\
& \text { and } \quad 0<\liminf _{n \rightarrow \infty} \beta_{n} \leq \limsup _{n \rightarrow \infty} \beta_{n}<1 .
\end{aligned}
$$

Then the sequence $\left\{x_{n}\right\}$ converges strongly to $z_{0} \in F(S) \cap E P(f)$, where $z_{0}=P_{F(S) \cap E P(f)}(I-V+$ $\gamma g) z_{0}$.

Proof Put $A=0$ and $B=\partial i_{C}$ in Theorem 10. Furthermore, for the bifunction $f: C \times C \rightarrow$ $\mathbb{R}$, define $A_{f}$ as in Lemma 14. Put $W=A_{f}$ in Theorem 10, and let $T_{r_{n}}$ be the resolvent of $A_{f}$ for $r_{n}>0$. Then we obtain that the domain of $A_{f}$ is included in $C$ and $T_{r_{n}} x_{n}=u_{n}$ for all $n \in \mathbb{N}$. Thus, we obtain the desired result by Theorem 10 . 


\section{Author details}

'Department of Mathematical and Computing Sciences, Tokyo Institute of Technology, Tokyo, 152-8552, Japan.

${ }^{2}$ Department of Applied Mathematics, National Sun Yat-sen University, Kaohsiung, 80424, Taiwan. ${ }^{3}$ Center for General Education, Kaohsiung Medical University, Kaohsiung, 80702, Taiwan.

\section{Acknowledgements}

The first author was partially supported by Grant-in-Aid for Scientific Research No. 23540188 from Japan Society for the Promotion of Science. The second and the third authors were partially supported by the grant Taiwan NSC 99-2115-M-110-007-MY3 and the grant Taiwan NSC 99-2115-M-037-002-MY3, respectively.

\section{Received: 25 May 2012 Accepted: 30 September 2012 Published: 17 October 2012}

\section{References}

1. Kocourek, P, Takahashi, W, Yao, J-C: Fixed point theorems and weak convergence theorems for generalized hybrid mappings in Hilbert spaces. Taiwan. J. Math. 14, 2497-2511 (2010)

2. Baillon, J-B: Un theoreme de type ergodique pour les contractions non lineaires dans un espace de Hilbert. C. R. Acad. Sci. Paris Ser. A-B 280, 1511-1514 (1975)

3. Kohsaka, F, Takahashi, W: Existence and approximation of fixed points of firmly nonexpansive-type mappings in Banach spaces. SIAM J. Optim. 19, 824-835 (2008)

4. Kohsaka, F, Takahashi, W: Fixed point theorems for a class of nonlinear mappings related to maximal monotone operators in Banach spaces. Arch. Math. 91, 166-177 (2008)

5. Takahashi, W: Fixed point theorems for new nonlinear mappings in a Hilbert space. J. Nonlinear Convex Anal. 11, 79-88 (2010)

6. Halpern, B: Fixed points of nonexpanding maps. Bull. Am. Math. Soc. 73, 957-961 (1967)

7. Wittmann, R: Approximation of fixed points of nonexpansive mappings. Arch. Math. 58, 486-491 (1992)

8. Takahashi, W: Convex Analysis and Approximation of Fixed Points. Yokohama Publishers, Yokohama (2000) (Japanese)

9. Kurokawa, Y, Takahashi, W: Weak and strong convergence theorems for nonspreading mappings in Hilbert spaces. Nonlinear Anal. 73, 1562-1568 (2010)

10. Hojo, M, Takahashi, W: Weak and strong convergence theorems for generalized hybrid mappings in Hilbert spaces. Sci. Math. Jpn. 73, 31-40 (2011)

11. Takahashi, W: Introduction to Nonlinear and Convex Analysis. Yokohama Publishers, Yokohama (2009)

12. Opial, Z: Weak convergence of the sequence of successive approximations for nonexpansive mappings. Bull. Am. Math. Soc. 73, 591-597 (1967)

13. Itoh, S, Takahashi, W: The common fixed point theory of single-valued mappings and multi-valued mappings. Pac J. Math. 79, 493-508 (1978)

14. Takahashi, W: Nonlinear Functional Analysis. Yokohama Publishers, Yokohama (2000)

15. Takahashi, W, Yao, J-C, Kocourek, K: Weak and strong convergence theorems for generalized hybrid nonself-mappings in Hilbert spaces. J. Nonlinear Convex Anal. 11, 567-586 (2010)

16. Takahashi, S, Takahashi, W, Toyoda, M: Strong convergence theorems for maximal monotone operators with nonlinear mappings in Hilbert spaces. J. Optim. Theory Appl. 147, 27-41 (2010)

17. Eshita, K, Takahashi, W: Approximating zero points of accretive operators in general Banach spaces. Fixed Point Theory Appl. 2, 105-116 (2007)

18. Aoyama, K, Kimura, Y, Takahashi, W, Toyoda, M: Approximation of common fixed points of a countable family of nonexpansive mappings in a Banach space. Nonlinear Anal. 67, 2350-2360 (2007)

19. Xu, HK: Another control condition in an iterative method for nonexpansive mappings. Bull. Aust. Math. Soc. 65 109-113 (2002)

20. Maingé, PE: Strong convergence of projected subgradient methods for nonsmooth and nonstrictly convex minimization. Set-Valued Anal. 16, 899-912 (2008)

21. Lin, L-J, Takahashi, W: A general iterative method for hierarchical variational inequality problems in Hilbert spaces and applications. Positivity. (to appear)

22. Rockafellar, RT: On the maximal monotonicity of subdifferential mappings. Pac. J. Math. 33, $209-216$ (1970)

23. Combettes, PL, Hirstoaga, SA: Equilibrium programming in Hilbert spaces. J. Nonlinear Convex Anal. 6, 117-136 (2005)

24. Aoyama, K, Kimura, Y, Takahashi, W: Maximal monotone operators and maximal monotone functions for equilibrium problems. J. Convex Anal. 15, 395-409 (2008)

25. Blum, E, Oettli, W: From optimization and variational inequalities to equilibrium problems. Math. Stud. 63, 123-145 (1994)

26. Takahashi, W, Wong, N-C, Yao, J-C: Two generalized strong convergence theorems of Halpern's type in Hilbert spaces and applications. Taiwan. J. Math. 16, 1151-1172 (2012)

27. Takahashi, S, Takahashi, W: Strong convergence theorem for a generalized equilibrium problem and a nonexpansive mapping in a Hilbert space. Nonlinear Anal. 69, 1025-1033 (2008)

28. Takahashi, W: Strong convergence theorems for maximal and inverse-strongly monotone mappings in Hilbert spaces and applications. J. Optim. Theory Appl. (to appear)

doi:10.1186/1687-1812-2012-181

Cite this article as: Takahashi et al.: Iterative common solutions for monotone inclusion problems, fixed point problems and equilibrium problems. Fixed Point Theory and Applications 2012 2012:181. 